\title{
Optical routing of nanospheres on plasmonic rails
}

\author{
Shengqi Yin, Fei He, Nicolas G. Green, Xu Fang* \\ School of Electronics and Computer Science, University of Southampton, Southampton SO17 1BJ, UK \\ *x.fang@soton.ac.uk
}

We demonstrate plasmonic nanoparticle routers that can guide and route nanospheres in a microfluidic channel in response to incident light. The results suggest a new method for next-generation microfabrication and sensing.

Recent convergence of plasmonics and microfluidics has opened up new research frontiers in the manipulation and detection of nanoparticles (for a recent review see [1]). Thanks to their unique optical, thermal and electrical responses to light illumination, plasmonic nanostructures promise novel functionalities that are often beyond the scope of traditional opto-microfluidics. This work demonstrates using metallic nanostrips to guide and route nanoparticles in a microfluidic channel. The device provides a high level of control over various nanoparticles by using nanostructures that are relatively easy to design and fabricate, with the potential for a new technology platform that will pay a significant role in next-generation microfabrication and sensing.
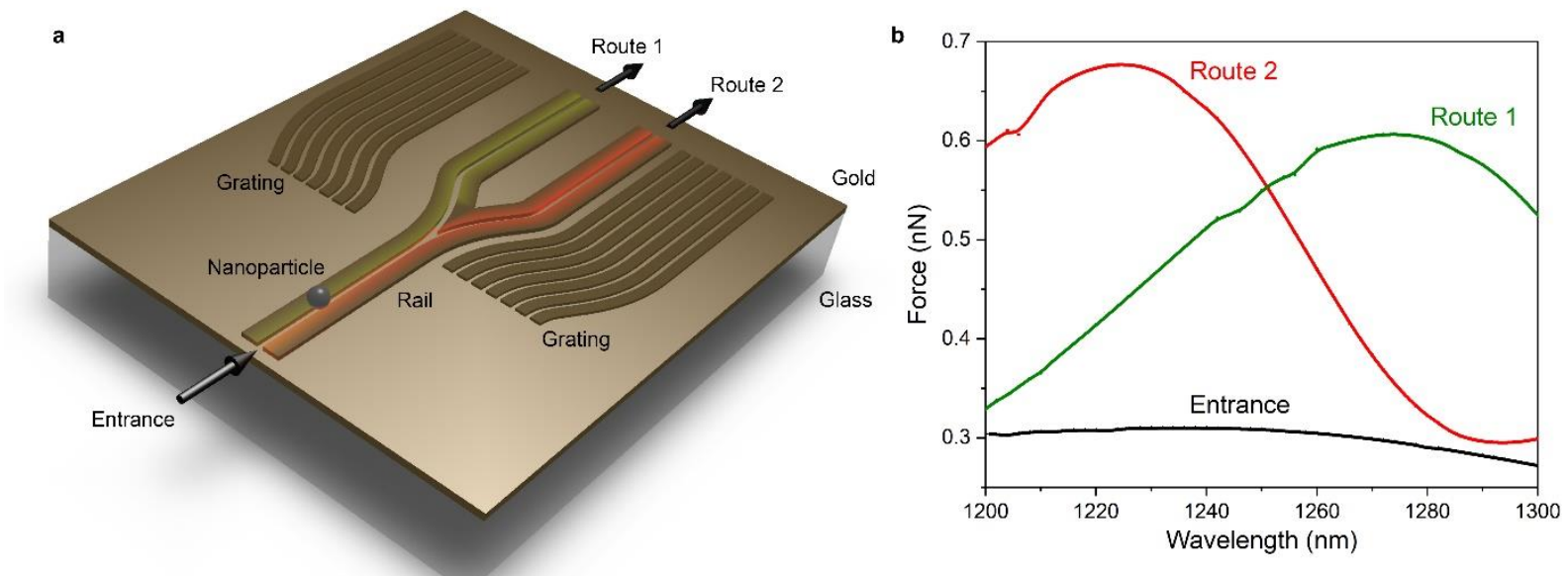

\begin{abstract}
Fig. 1 (a) Schematic of the plasmonic nanoparticle router. Nanoscale rails and gratings are fabricated on top of a thick gold film coated on a glass substrate. The whole device is at the bottom of a microfluidic channel, which is under light illumination from the top. While the rail close to the entrance supports plasmonic resonance over a broad wavelength range (as depicted by the coexistence of both green and red colours), the rail in Routes 1 and 2 is far more selective in the resonance wavelength (as depicted by the two different colours). By controlling the illumination wavelength, a nanoparticle entering the microfluidic channel will follow either Route 1 or Route 2 in its trajectory. (b) Numerically simulated plasmonic force on the nanoparticle along the rail. The nanoparticle is at the centre of the rail and is $10 \mathrm{~nm}$ above its top surface. The force shows very weak wavelength dependence close to the entrance. By comparison, both the peak strength and the wavelength dependence are far stronger in Routes 1 and 2 . These differences, induced by the gratings, are utilised for optical routing.
\end{abstract}

It is generally accepted that plasmonic resonances create strong and localised optical fields that allow for tight trapping of nanoparticles. A key aspect of this work is to use a variety of nanostrips to control the strength, location and wavelength of the resonance. Figure 1 shows the working principle through an example device design and the optical force. The device is at the bottom surface of a microfluidic channel in which nanoparticles are carried forward by a gentle flow of water. The device contains many nanostrips that are grouped into the rail (for trapping and guiding the nanoparticles) and the two gratings (for wavelength selection). The rail close to the entrance of the device, due to the absence of any grating at either of its side, supports plasmonic resonance over a broad wavelength range. Immediately after the divergence, a grating is placed close to the rail by its side. The two gratings, due to their difference dimensions, provide constructive interference of light at the rail at two different wavelengths. As illustrated in Fig. 1a, even though the whole device is under light illumination, a designated wavelength only excites plasmonic resonance along a particular route along which the nanoparticle flows.

As an example, Fig. 1b shows the numerically simulated force exerted by light on a polycarbonate nanosphere (diameter $220 \mathrm{~nm}$, refractive index 1.58). Only the force vertical to the device surface (i.e. the force that pins down the nanosphere) is shown here. The influence of the grating on the magnitude and the wavelength dependence of the force is profound. In addition to Fig. $1 \mathrm{~b}$, forces exerted on several different nanoparticles in different directions have been calculated. These results confirm that nanoparticles can be routed between nanoscale channels by controlling the wavelength of incident light.

[1] D. Gao, W. Ding, M. Nieto-Vesperinas, X. Ding, M. Rahman, T. Zhang, C. Lim, and C-W Qiu, " Optical manipulation from the microscale to the nanoscale: fundamentals, advances and prospects," Light: Science \& Applications 6, e17039 (2017). 